\title{
Front-End Electronics of the Compact High Energy Camera
}

\author{
S A Leach ${ }^{\mathrm{a}, *}$, J S Lapington ${ }^{\mathrm{a}}$, D Ross ${ }^{\mathrm{a}}, \mathrm{J}_{\text {Thornhill }}^{\mathrm{a}}, \mathrm{C}_{\text {Duffy }}{ }^{\mathrm{a}}$,

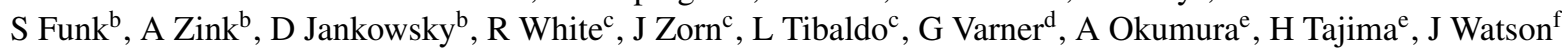 \\ ${ }^{a}$ University of Leicester, UK \\ ${ }^{b}$ Friedrich-Alexander-Universität Erlangen-Nürnberg (FAU), ECAP \\ ${ }^{c}$ MPI für Kernphysik \\ ${ }^{d}$ University of Hawaii \\ ${ }^{e}$ Nagoya University \\ ${ }^{f}$ University of Oxford
}

\begin{abstract}
The Compact High Energy Camera is a focal plane camera designed for two mirror Schwarzschild-Couder design imaging air Cherenkov telescopes such as the SST-2M variants on the Cherenkov Telescope Array. It utilises a 2048-pixel array of silicon photomultipliers arranged in thirty-two $8 \times 8$ pixel $^{2}$ tiles. Each detector tile is instrumented with a front-end electronics module designed to provide single photon counting with sub-nanosecond timing, full-waveform digitisation and event triggering capabilities based around TARGET ASICs. Performance results including triggering, digitiser noise, signal crosstalk, linearity and dynamic range from initial laboratory tests have been collated and are presented.
\end{abstract}

Keywords: CHEC, TARGET, CTA, front-end electronics, silicon photomultipliers, readout, SST-2M, full-waveform readout

\section{INTRODUCTION}

The Compact High Energy Camera (CHEC) is an ultrasensitive single-photon counting camera being developed to image Cherenkov light flashes, peak $\lambda \approx 400 \mathrm{~nm}$, generated during gamma-ray initiated particle showers. CHEC-S is the second prototype version camera and uses silicon photomultiplier (SiPM) pixels whereas CHEC-M, the first prototype, is based on multi-anode photomultipliers. The development and characterisation of CHEC-M, in the laboratory and on-telescope, validated the CHEC concept [1] and enabled several technical improvements to contribute to a new design. CHEC-S is currently undergoing full characterisation with on-telescope observations being planned for spring 2019. This article describes the new Front-End Electronics (FEE) modules developed and optimised to instrument imaging air Cherenkov telescope cameras equipped with SiPM photodetectors.

\section{FRONT-END ELECTRONICS}

The new CHEC-S FEE camera modules (figure 1; schematic ${ }^{29}$ layout, figure 2 photograph) each accommodate 64 identical channels, enabling 2048 discrete pixels to be read out by $32^{30}$ modules in each camera. CHEC-S uses $3 \times 3 \mathrm{~mm}^{2} \mathrm{SiPMs}^{31}$ grouped in fours resulting in $6.4 \mathrm{~mm}^{2}$ pixels. Each signal chan- ${ }^{32}$ nel comprises a fast analogue shaper to optimise the pulse char- ${ }^{33}$ acteristics $\left(\approx 8 \mathrm{~ns}\right.$ FWHM) for the high-speed digitiser, and $\mathrm{a}^{34}$

\footnotetext{
${ }^{*}$ Corresponding author

Email address: drleach@physicsresearch.co.uk (S A Leach)
}

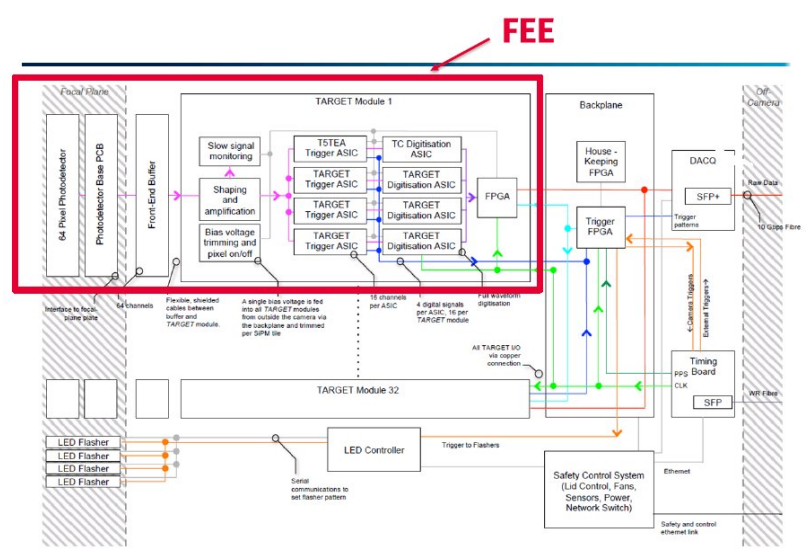

Figure 1: CHEC-S schematic layout with the FEE module section indicated.$$
\text { low }
$$

OPTIMISATION. The updated FEE module design, led by the University of Leicester in the UK, includes physically separate analogue and digital functionality, to minimise crosstalk, split trigger and digitiser operation, and optimised electromagnetic shielding. Power conditioning for the FEE and silicon photomultiplier array has been moved to a separate PCB which also houses additional housekeeping monitoring functionality (figure 3). Module temperature is stabilised $\left(\approx 23^{\circ} \mathrm{C}\right)$ by circulating cooled air inside the camera with excess heat extraction via 


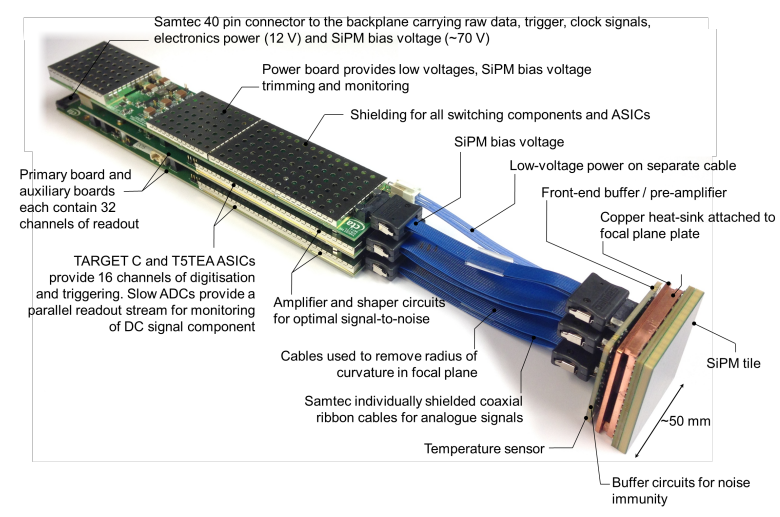

Figure 2: One of 32 identical FEE modules for CHEC-S. Each has 64 independent pixel channels optimised for Cherenkov flashes with full-waveform digitisation of the $8 \mathrm{~ns}$ shaped event pulses.

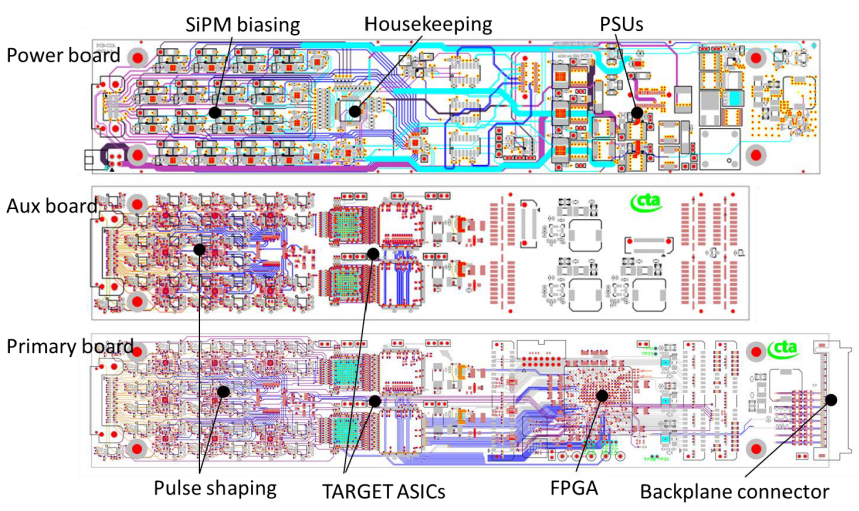

Figure 3: The layout of the three tiers of board, each optimised to minimise noise and crosstalk between channels. an external water chiller.

TARGET CHIPSET. Conditioned event pulses are fed to the latest generation TARGET chipset which consists of a high ${ }^{75}$ speed 1GS/s 12-bit digitiser (TARGET C) in parallel with a trig- ${ }_{76}$ ger chip (T5TEA), acting on summed groups of $2 \times 2$ channels known as "super-pixels". The latter provides a first level overthreshold trigger which is processed by a pattern-matching al- ${ }_{79}$ gorithm to generate a camera trigger for a validated event. Valid ${ }_{80}$ event determination results in a full readout of the camera, each ${ }_{81}$ of the FEE modules providing 64 digitised waveforms of typically 96 ns duration, and can be elongated if required.

\section{TARGET MODULE FEATURES.}

- 64 signal channels

- 4 x T5TEA ASIC (trigger) - 16 LVDS trigger signals per module

- 4 x TARGET C ASIC (sampling) at $1 \mathrm{GSa} / \mathrm{s}$

- $4 \mu$ s $(16 \mu$ s possible) deep ring buffer

- Data via simple User Datagram Protocol (UDP)

- Firmware upgradable via UDP

- Slow signal path for pointing and Night Sky Background 91 analysis

- ns trigger timing on module

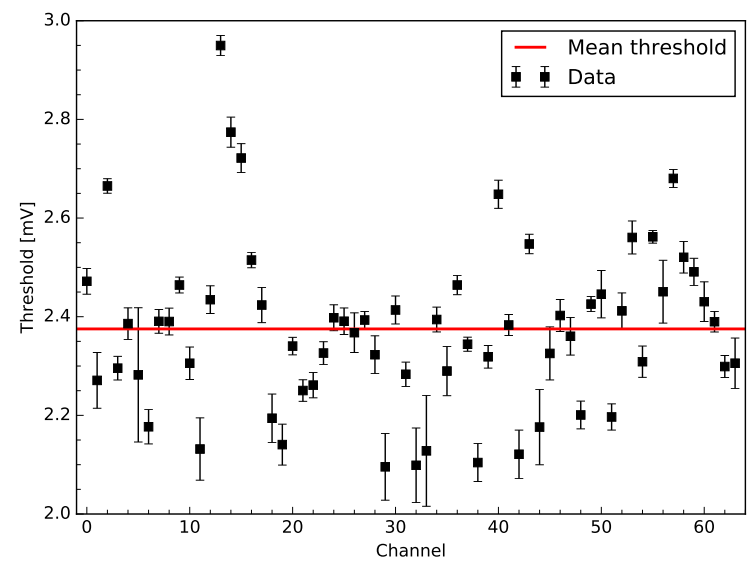

Figure 4: Trigger performance results across all channels of a module measure minimum trigger of $(2.38 \pm 0.33) \mathrm{mV}$. Red line is mean threshold.

- Tunable offset (voltage pedestal) for each channel (trigger and sampling)

- 3 PCB stack (Primary, Auxiliary, Power)

- $16 \mathrm{HV}$ bias groups

FULL-WAVEFORM CAPTURE ADVANTAGES. A major feature of the CHEC design is the ability to digitise and read out a complete event signal with 1 ns resolution. This has many advantages over peak detect counterparts: The signal dynamic range is extended from $\approx 500$ to over 2000 photo electrons (pe) by using pulse reconstruction of the saturated waveforms, precise (per pixel) event arrival time spanning the typical 100 ns readout window (essential for transit tracking high-energy gamma-ray events), background event rejection and can be used for advanced image cleaning.

\section{TRIGGER PERFORMANCE}

The FEE module triggers on an analogue sum of four channels (super-pixels) and is configured through several T5TEA parameters which are established during module calibration, performed at ECAP, Erlangen. These parameters include a per channel voltage pedestal, a per trigger-group DC offset and trigger width trim, and a signal threshold level. Figure 4 illustrates the minimum trigger threshold for each channel with a typical triggering performance of $<3 \mathrm{mV}$ ( $0.6 \mathrm{pe})$, compared to the previous TARGET T7/T5 results of 50/20 mV respectively [3]. Trigger noise is measured at $0.33 \mathrm{mV}(0.1$ to $0.15 \mathrm{pe})$ for all channels with sampling on $\left(23^{\circ} \mathrm{C}\right.$ ambient $)$.

\section{DIGITISING PERFORMANCE}

TARGET C digitiser noise has been measured on-module (without the photodetectors or buffer-preamplifier attached) using an external trigger. A unique electronic pedestal, value per dow. Each ASIC channel used 4096 storage cells (of the 16384 available) and has a dynamic range of $1.9 \mathrm{~V}$. Typical measured storage cell/sample, is subtracted from the 96 ns readout win- 
noise illustrated in figure 5 is 1.2 ADC counts $\approx 0.6$ to $1 \mathrm{mV}_{117}$ (RMS) (equivalent to $\approx 0.2$ pe) across the whole module.

\section{SIGNAL CROSSTALK}

Signal crosstalk between channels has been measured for both the primary and auxiliary boards ( 32 channels on each). By injecting a known amplitude signal pulse into a channel and measuring coincident crosstalk levels (pedestal calibrated) on the 31 corresponding channels illustrates all channels have $<1 \%$ crosstalk (figure 6.

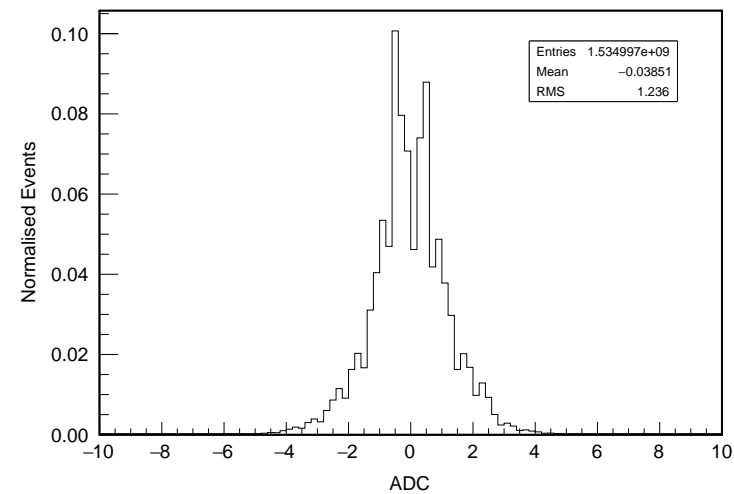

Figure 5: Pedestal subtracted baseline illustrating digitiser noise across the whole module of 1.2 ADC counts RMS (without preamplifier).
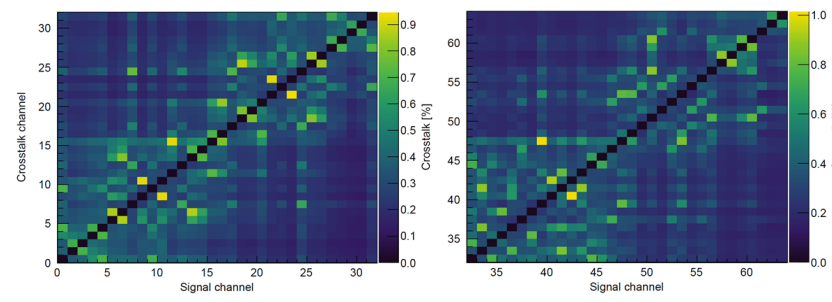

Figure 6: Signal crosstalk between module channels presented for channels 031 (primary board) and 32-63 (auxiliary board). Black pixels are corresponding input channels so no measurement expected.

\section{CAlibration}

Each FEE module requires a calibration process to compencur along each signal path. Calibration normalises and lin- ${ }_{136}$ earises the response of each pixel channel. This process re- ${ }_{137}$ quires generation of Transfer Functions (TF) to correct for the non-linearity in the ASIC switched-capacitor response to signal amplitude. The TF, along with pedestal subtraction, is then used ${ }^{138}$ to reconstruct the charge. For TARGET, the TF also depends on the relative signal position in the storage array, plus AC effects ${ }_{140}$ ( signal $\approx 80 \mathrm{MHz}$ ) of TF below ASIC bandwidth $(>500 \mathrm{MHz}){ }^{141}$ Therefore, the adopted approach for TARGET C is to measure ${ }^{142}$ the AC TF as follows: Contain module in a temperature con- ${ }_{144}^{143}$ trolled chamber; inject signal-like pulse of known amplitude; ${ }_{145}$ read ADC value of peak position (storage cell) and then step increase the amplitude to generate a cell-dependant lookup table. This procedure results in $\approx 100 \mathrm{MB}$ of TF data per module which is stored and applied offline and used to calibrate camera image data.

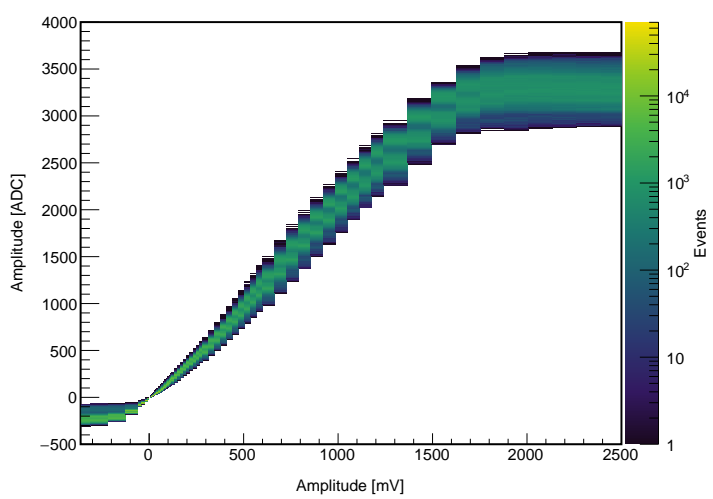

Figure 7: AC transfer functions generated for all channels on one module at a constant temperature.

Figure 7 illustrates typical TFs measured across a complete module (64 channels x 4096 storage cells). These TFs are calculated and stored on a per-module basis and subsequently applied to camera acquired data for detailed calibrated analysis.

\section{CONCLUSION}

A new TARGET C/T5TEA ASIC based module has been designed for use with the SiPM based CHEC prototype camera. With a measured performance of: Trigger $(2.38 \pm 0.33) \mathrm{mV}$ and digitiser noise of $<1 \mathrm{mV}$ RMS $(\approx 0.2 \mathrm{pe}), 32$ calibrated modules have been integrated into the complete camera system at MPIK, Heidelberg. CHEC group members are currently performing detailed characterisation in the laboratory and are preparing for the first on-telescope trials in spring 2019.

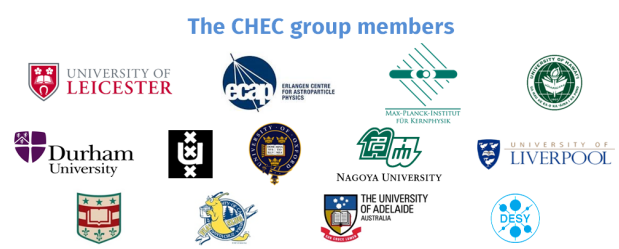

We gratefully acknowledge financial support from the agencies and organisations listed here: www.ctaobservatory.org/consortium_acknowledgments

\section{REFERENCES}

[1] J. Zorn, et al, Characterisation and Testing of CHEC-M - a camera prototype for the Small-Sized Telescopes of the Cherenkov Telescope Array, Instrumentation and Methods for Astrophysics 904 (2018) 44-63. doi:10.1016/j.nima.2018.06.078

[2] S. Funk, et al, TARGET: A Digitizing And Trigger ASIC For The Cherenkov Telescope Array, AIP Conference Proceedings 1792 (2017) 080012. doi:10.1063/1.4969033 
[3] A. Albert, S. Funk, T. Kawashima, M. Murphy, A. Okumura, R. Quagliani, L. Sapozhnikov, H. Tajima, L. Tibaldo, J. Vandenbroucke, G. Varner, T. Wu, TARGET 5: a new multi-channel digitizer with triggering capabilities for gamma-ray atmospheric Cherenkov telescopes, Astroparticle 150 Physics 92 (2017) 49-61. doi:10.1016/j.astropartphys.2017.05. $151 \quad 003$ 